\title{
Experiencia de trabajo interdisciplinario e interinstitucional en la residencia de Salud Pública Veterinaria en centros de salud de la Cuarta Zona Sanitaria de la Zona Andina, Rio Negro, República Argentina
}

\author{
${ }^{1}$ Santos, K.N.; ${ }^{2}$ Mujica, G.B.
}

${ }^{1}$ Residente en Salud Pública Veterinaria, Ministerio de Salud de Rio Negro - Facultad de Ciencias Veterinarias UNLPAm

${ }^{2}$ Coordinador de la Residencia en Salud Pública Veterinaria Zona Andina, Ministerio de Salud de Rio Negro.

Laprida 240 (8500) Viedma, Río Negro

Contacto: karinanataliasantos@gmail.com

Fecha de recepción de originales: 20/08/2017

Fecha de aceptación para publicación: 3/11/2017

\section{Introducción}

La Residencia en Salud Publica Veterinaria (RSPV) y la Especialización en Salud Publica Veterinaria (ESPVET) dictadas en cooperación entre el Ministerio de Salud de Rio Negro y la Facultad de Ciencias Veterinaria de la UNLPam son de las pocas ofertas académicas existentes en el país para aquellos veterinarios que quieren orientarse, formarse y trabajar en el campo de la Salud Publica Veterinaria.

El objetivo es, a través de la capacitación en servicio, formar Médicos Veterinarios capaces de desempeñarse en el seno de una comunidad, con una formación científica, metodológica e instrumental que les permita abordar los problemas que signifiquen un riesgo para la salud y bienestar de las personas. 
Para ello, se integra un proceso de formación práctica y teórica desarrollada en la forma de residencia en los servicios de salud pública veterinaria del Ministerio de Salud de la provincia de Rio Negro con la formación académica y el desarrollo de una tesina bajo la tutela de la Facultad de Ciencias Veterinarias de la UNLPam, conducente a la obtención del título de Especialista en Salud Publica Veterinaria, con acreditación de CONEAU.

El proceso formativo teórico y práctico abarca todas las áreas que involucran a la Salud Pública Veterinaria con especial referencia a control de zoonosis, inocuidad de alimentos y saneamiento ambiental.

El entrenamiento incluye desarrollar una experiencia interdisciplinaria e interinstitucional dentro del equipo de trabajo de los Centros de Salud urbanos y rurales de la zona andina, de la provincia de Rio Negro, a los efectos de posibilitar un accionar integrado con la comunidad y promover aspectos vinculados a la protección de los alimentos, el saneamiento básico y la prevención y control de las zoonosis prevalentes desde una perspectiva de atención primaria de la salud bajo el nuevo paradigma "un mundo, una salud".

\section{Metodología de trabajo}

Desde el ingreso del residente al servicio de Salud Ambiental del Ministerio de Salud, luego de un concurso público abierto, y durante los 3 años de la RSPV, el Médico Veterinario asiste una vez por semana a un centro de salud urbano de la ciudad de San Carlos de Bariloche.

Existen once Centros de Salud dependientes del Hospital complejidad VI de San Carlos de Bariloche, que cuentan con recursos humanos variables, según su complejidad, pudiendo incluir Agentes Sanitarios, Médicos Generalistas, Residentes de Medicina General, Odontólogos, Nutricionistas, Trabajadores Sociales, Psicólogos, Enfermeras, Mucamas y Administrativos.

La población urbana que atienden, tiene características socioeconómicas y culturales muy variadas, presentando desde condiciones básicas de saneamiento aceptables, hasta condiciones de extrema precariedad de saneamiento sobre todo en las zonas de la ciudad.

Los residentes realizan pasantías y rotaciones en Centro de Salud rurales de la Zona Andina de la provincia de Rio Negro. Los 
Centros de Salud Rurales, en los cuales participa la RSPV, son los pertenecientes al paraje Villa LLanquin y paraje Villegas-Manso.

El paraje Villegas-Manso depende del Hospital El Bolsón y allí se acciona en conjunto con el equipo de trabajo de la Unidad Regional de Epidemiologia y Salud Ambiental de la Zona Andina Sede El Bolsón. En el paraje Villa LLanquin se trabaja en equipo con un Agente Sanitario local, personal del DAPA (Departamento de Actividades Programadas) de la ciudad de San Carlos de Bariloche y la Residencia de Medicina General de Bariloche. En dicho paraje la población es mayoritariamente rural, distribuida de manera difusa a lo largo de la geografía y solo un bajo porcentaje está concentrado alrededor del mismo.

La forma de trabajo en los Centros de Salud, es organizada, planificando actividades interdisciplinarias e interinstitucionales, con el equipo de salud, con las instituciones barriales, con organismos gubernamentales como INTA, Universidad del Comahue de Rio Negro, Colegio Médico Veterinario de RN, entre otros, buscando mejorar aspectos básicos de saneamiento, control de las zoonosis y protección alimentaria. Asimismo, se trabaja en situaciones puntuales asociadas a las necesidades básicas insatisfechas de la comunidad, en poblaciones especialmente vulnerables y de riesgo.

Se plantean metas de trabajo para el MV RSPV. La primera meta es la inserción del mismo, dentro del equipo de Salud. Para ello se requiere confeccionar un diagnóstico de situación de la comunidad en cuestión, con propósito de identificar factores de riesgo y priorizar los problemas para en un futuro trabajar sobre ellos.

Como segunda meta se plantea la intervención propiamente dicha, dentro de la comunidad. Y como tercera y última meta la comunicación de lo trabajado en el período de la residencia.

Actualmente la RSPV con sede en Bariloche cuenta con dos residentes de primer año y un residente de segundo año, formando parte de los equipos de los Centros de Salud San Francisco III, Ojos de agua y 2 de abril.

Durante el transcurso de la Residencia, el MV residente, es supervisado en sus actividades desde el área de Salud Ambiental por su Coordinador. El equipo de trabajo del Centro de Salud también supervisa la inserción y el desempeño del residente. 
Algunos ejemplos de las actividades específicas que se realizaron durante el año 2016 y que continúan en el presente año, en los Centros de Salud urbanos y rurales son:

Protección alimentaria:

- Talleres de buenas prácticas de manipulación de alimentos destinadas a comedores escolares y feriantes.

- Prevención de enfermedades alimentarias como SUH, con intervenciones en jardines de infantes y carnicerías.

Saneamiento Básico:

- Salidas a terreno con los agentes sanitario y residente de medicina general del equipo de salud para conocer el territorio;

- Participación en censos realizados en las tomas pertenecientes al radio del Centro de Salud, con el objetivo de conocer a la población;

- Abordaje de problemas de saneamiento barriales tales como: micro basurales, derrame de líquidos cloacales a la calle, control de cloración.

- Talleres de lavado de manos en escuelas, jardines y salas de espera del Centro de Salud.

Control de Zoonosis:

- Controles ecográficos en pacientes con Hidatidosis;

- Catastros ecográficos en niños escolares dentro del programa Salud Escolar y Programa Provincial de Control de Hidatidosis.

- Vacunación antirrábica y desparasitación para el día del animal;

- Actividades de promoción y prevención de enfermedades zoonóticas mediante la entrega de afiches y folletos en diferentes instituciones barriales, escuelas y jardines.

- Talleres y charlas en escuelas, jardines y salas de espera a partir de actividades programadas que surgen de las fechas claves en salud, tales como el día del Lavado de Manos, día de Lucha contra del SUH, etc.

- Entrega de carnet y antiparasitarios, además de la concientización sobre tenencia responsable de mascotas durante todo el año. Charlas de tenencia responsable en escuelas.

Participación en otros temas vinculados a la Salud:

- Participación en talleres de educación sexual, alimentación saludable, taller de cepillado de dientes, etc. 
- Participación en jornadas de atención primaria.

- Participación en la organización del congreso de Medicina General 2016.

\section{Conclusiones}

En abril de 2010, OMS, FAO y OIE emitieron el documento D-10855 distribuyendo responsabilidades y coordinación de actividades mundiales para abordar los riesgos sanitarios en la interfase humana-animal-ecosistémica. Actualmente expresada en el concepto de Un Mundo, Una Salud.

La integración de los servicios de SPVET y Salud Publica tradicional, incluyendo la articulación entre las residencias en SPVET, Medicina General y Salud Mental Comunitaria y otras organizaciones gubernamentales y no gubernamentales, se encuentra en esa línea de trabajo.

En nuestro ámbito de trabajo cotidiano, la RSPV permite lograr y fortalecer vínculos interdisciplinarios e interinstitucionales en el campo de la Salud Pública, permitiendo así, promover el bienestar de las personas previniendo problemas que signifiquen un riesgo para la salud en la comunidad.

El trabajo que realiza un Médico Veterinario de la RSPV en el equipo de Salud, permite sensibilizar a la población en temas de promoción y prevención de enfermedades, trabajando más estrechamente con la misma y conociendo sus realidades.

La tarea de los residentes en SPVET, asimismo, enriquece a los equipos de salud, acerca a la población al Centro de Salud al desarrollar temas que son de interés comunitario y ayuda a mantener vínculos con la comunidad que se traducen en menor riesgo de enfermar. 
Participación en los festejos del día del niño, entrega de antiparasitarios y sensibilización en Tenencia responsable de mascotas. Centro de Salud "Ojos de Agua". Agosto 2016.

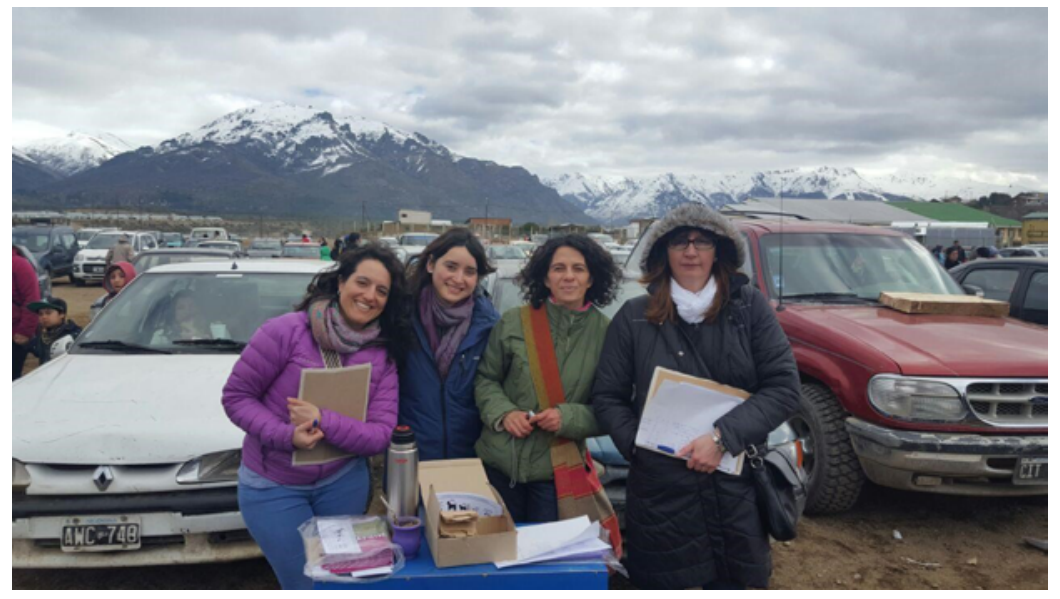

Taller de Lavado de Manos en Jardín. Barrio San Francisco. Julio 2017.

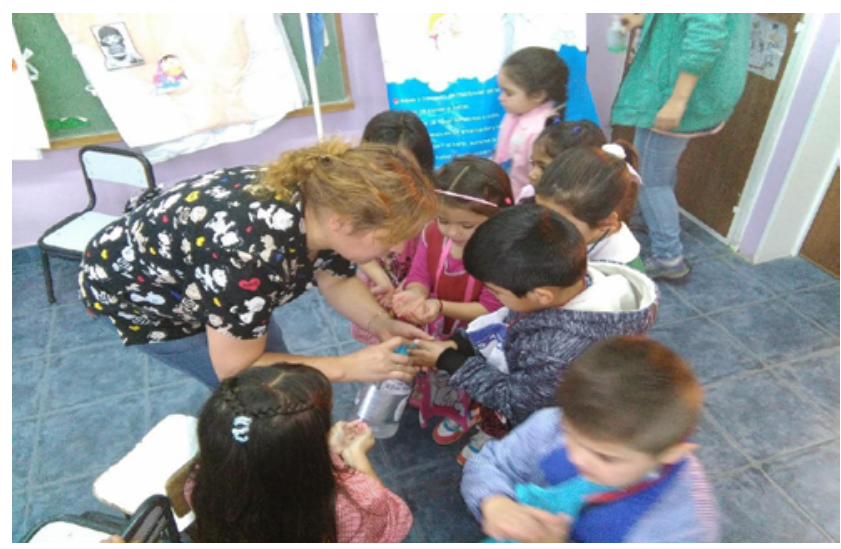

